\title{
Introduction - Etangs : paysages, pays et territoires
}

Introduction - Ponds: landscapes and territories

Einleitung - Weiher : Raum, Landschaft und Territorium

\section{Denis Mathis}

\section{OpenEdition}

\section{Journals}

Electronic version

URL: http://journals.openedition.org/rge/5711

DOI: $10.4000 /$ rge.5711

ISSN: 2108-6478

\section{Publisher}

Association des géographes de l'Est

Printed version

Date of publication: 22 April 2016

ISSN: 0035-3213

\section{Electronic reference}

Denis Mathis, «Introduction - Etangs : paysages, pays et territoires », Revue Géographique de l'Est

[Online], vol.56 / n¹-2 | 2016, Online since 05 September 2016, connection on 23 September 2020.

URL : http://journals.openedition.org/rge/5711 ; DOI : https://doi.org/10.4000/rge.5711

This text was automatically generated on 23 September 2020.

Tous droits réservés 


\title{
Introduction - Etangs : paysages, pays et territoires
}

\author{
Introduction - Ponds: landscapes and territories \\ Einleitung - Weiher : Raum, Landschaft und Territorium
}

\section{Denis Mathis}

1 Malgré déjà de nombreux travaux et études menés ces dernières années (Touchart (dir.), 2007 ; Bénarrous, 2009 ; Bédoucha, 2011; Bartout et Touchart, 2013 ; Bartout, 2015a ; Touchart, Bartout et Motchalova(dir.), 2015), l'objet géographique que constitue l'étang et les territoires d'étangs reste encore méconnu ; inventaire, usages, gestion, fonctionnalités, aquaculture, activités de tourisme et de loisirs, valeurs écologiques, biodiversités, paysages, territoires ruraux, autant de thèmes qui gravitent autour de cet objet et qui permettraient de mieux appréhender le potentiel d'une «France aux 250000 étangs » (Bartout, 2015b). Territoires ruraux marginaux, longtemps déclinants, les pays d'étangs et leurs paysages constituent aujourd'hui une ressource et une opportunité pour des formes de renaissance rurale et de projets territoriaux comme l'ont souligné St. De Carrara et Y.-F., Le Lay (2015) pour les Dombes dans l'Ain ou encore D. Mathis (2014) pour le Pays des Étangs en Lorraine. Alors que les paysages et les territoires ruraux ont connu une évolution rapide au cours des dernières décennies, les paysages d'eau apparaissent comme immuables, et sont ainsi perçus comme des «paysages authentiques» pour des populations en recherche de nature. À l'image négative du XIXe siècle, renvoyée par les "eaux mortes et stagnantes», s'oppose aujourd'hui celle apaisante et sereine de paysages immobiles presque intangibles. C'est cette représentation qui conforte l'attractivité touristique et de loisirs de ces étendues d'eau. Aussi pour ces espaces, il devient nécessaire de concilier tout autant la gestion de la ressource constituée par les paysages d'étangs que de leur assurer une attractivité nouvelle. Cette gouvernance territoriale des acteurs locaux, des gestionnaires d'étangs ou de domaines d'étangs, ne doit pas être confondue avec la demande de gestion institutionnelle et réglementaire.

2 Les étangs, écosystèmes nés de l'activité humaine, constituent un objet d'étude privilégié de l'interface Homme/Nature et sont pourtant encore insuffisamment 
étudiés. Ces écosystèmes fragiles ont été façonnés et aménagés aux dépens des zones humides et des cours d'eau. Ils ont été intégrés par l'Homme au sein de systèmes productifs (pêche, industrie, transports, carrières en eau...) aujourd'hui souvent obsolètes ou partiellement déclassés. La perte partielle ou totale des fonctionnalités traditionnelles des étangs a rompu les liens systémiques permettant leur équilibre relatif. Façonnées et entretenues par l'activité des hommes, ces étendues d'eau, parce qu'elles sont convoitées, justifient aujourd'hui une politique d'entretien, voire de conservation, qui aboutit à des empilements de zones de préservation avec, comme conséquences, des "mises sous cloche» comme l'ont souligné E. Chiffre et D Mathis (2015) pour le Morvan et la Lorraine. Ces dernières semblent d'ailleurs en contradiction avec la Directive Cadre Européenne sur l'Eau qui cherche à rétablir les continuités écologiques et finalement considère le patrimoine lentique comme un obstacle à la bonne gestion des cours d'eau, au rétablissement des corridors écologiques et à la qualité des eaux. Ainsi, cette directive s'est accompagnée de multiples travaux de renaturation destinés à maintenir ces cours d'eau dans un état de naturalité supposé et parfois idéalisé (suivant une vision des auteurs romantiques ou réalistes du XIX ${ }^{\mathrm{e}}$ transposée aujourd'hui par les gestionnaires de l'eau). La faible prise en compte de la valeur historique, sociale, patrimoniale ou culturelle des étangs et des spécificités des pays d'étangs, se traduit par des stratégies de gestion conduisant à l'effacement des étangs, faisant table rase de siècles d'aménagement anthropique et niant un patrimoine lentique qui dépasse le simple objet spatial de l'étang.

Ce constat doit permettre de s'interroger sur «la vie et la mort» des étangs. Leur évolution naturelle, surtout en l'absence d'entretien, tend vers l'atterrissement, le comblement ; seule l'activité humaine, qu'elle soit productive ou conservatrice, permet de pérenniser ces étendues d'eaux mortes. Aussi, quelles que soient les échelles, de l'étang au pays d'étangs, la médiance nécessaire entre la préservation et la consommation de ce type d'espace sous-tend le concept d'utilisation rationnelle. Les articles rassemblés dans ce numéro de la Revue Géographique de l'Est présentent les interrogations sur les paysages et les pays d'étangs, visitant à plusieurs échelles les dynamiques de ces territoires. Les contributions construisent le plus souvent leur démarche sous le prisme de la géohistoire, à l'échelle des paysages et des pays d'étangs, ces derniers présentant des dynamiques, des transitions et des trajectoires fluctuantes de leur identité, de leur perception, de leur gestion mais aussi de l'essor et de leur déclin. Les auteurs évoquent également la mutation fonctionnelle des étangs, des paysages et des pays d'étangs à l'aune du concept d'utilisation rationnelle et des enjeux de gestion notamment au sein de milieux urbains et périurbains ainsi que les enjeux de préservation des étangs et par la même des pays d'étangs.

Dans un premier temps, Pascal Bartout et Laurent Touchart interrogent la notion de limnicité pour caractériser l'empreinte spatiale et sociétale des plans d'eau. Les auteurs développent leur questionnement à partir de l'étude de deux États : l'Estonie et la France, en fonction des critères de «limnicité » et «limnicité étendue». Les divergences soulignent les limites de ces notions dont l'approche est trop partielle pour les milieux lentiques. Les auteurs préconisent d'accompagner la limnicité d'autres indicateurs pertinents destinés à faciliter la compréhension de la place de l'Homme dans ces milieux pour établir la recherche d'un équilibre Homme/Nature. La richesse des étangs, créations anthropiques, repose tout autant sur leur potentiel écologique à réévaluer que sur leur histoire, leur économie, leurs rôles social et sociétal. Considérer les étangs en tant que patrimoine matériel et immatériel doit permettre de faciliter la 
mise en œuvre des périmètres de gestion à diverses échelles. Il est donc nécessaire d'intégrer à la réflexion les disparités spatiales, économiques, fonctionnelles ou encore écologiques pour pouvoir établir une politique de gestion qui s'inscrit encore dans le discernement de ce milieu.

5 À l'échelle nationale et suivant une méthode associant géohistoire et statistique, Pascal Bartout interroge les dynamiques des territoires d'étangs. Il en souligne les trajectoires multiples et en constantes réécritures depuis l'épisode révolutionnaire jusqu'à nos jours. Cette approche lui a permis de souligner qu'au-delà du concept d'hydroécorégions destiné à la mise en œuvre de la Directive Cadre Européenne sur l'Eau (outil conceptuel établi suivant des critères combinant la géologie, le relief ou le climat considérés comme les déterminants des écosystèmes d'eau courante), les territoires lentiques sont des géosystèmes centrés sur les étangs. Ils sont liés à des paramètres sociaux, économiques et surtout historiques qui ont façonné une " image stagnustre ", voire une identité autour des étangs. En emboîtant les échelles, Pascal Bartout a souligné la prégnance et la permanence des territoires d'étangs au sein de trois régions (Centre-Val-de-Loire, Pays-de-la-Loire et Limousin). Cette continuité, malgré des " accidents historiques » rappelle les évolutions des demandes sociétales permettant de définir des critères de fonctionnement, de gestion, de répartition des territoires d'étangs. Ainsi, peut-on évoquer un autre outil conceptuel : la limnorégion.

6 À l'échelle de la Lorraine, Denis Mathis et Anne Mathis retracent sur un temps long les dynamiques des pays d'étangs. Ils soulignent l'important patrimoine lentique aménagé dans les fonds de vallées du massif vosgien et de ses marges gréseuses ou dans les dépressions argilo marneuses aux chevelus hydrographiques denses du relief de côte. Dans les deux cas, ces aménagements ont permis l'essor d'une activité agropiscicole établie en particulier sur de grands domaines aquacoles. Ils ont façonné un "terroir» d'étangs qui s'est juxtaposé au système d'openfield. Le déclin de ces domaines piscicoles s'est accompagné du développement de nouveaux usages de la ressource en eau. Désormais ces territoires d'eau constituent des éléments centraux des parcs naturels régionaux lorrains, ou sont au cœur de projets territoriaux. Ils concilient activités piscicoles résilientes, loisirs et tourisme et préservation environnementale avec parfois des conflits d'usage. Cependant à la périphérie des aires urbaines se mettent en place de nouveaux terroirs d'étangs par reconversion/réhabilitation d'anciennes gravières. Ces nouveaux « étangs » se trouvent eux aussi au cœur d'usages nouveaux et de compromis. Longtemps la valeur environnementale de ces étangs a été sous-évaluée avant d'être proposée pour intégrer les projets de trames vertes et bleues. Cette reconnaissance écologique devra concilier également les demandes de « paysages d'eau » des populations urbaines. Les pays d'étangs lorrains permettent de souligner les évolutions des assolements des anciens et nouveaux terroirs d'étangs.

7 Anatole Danto présente les usages traditionnels de l'eau en Lusace, territoire de la minorité sorabe dans le nord-est de l'Allemagne. L'hydromorphie de cette région située à cheval sur les Länder de Saxe et du Brandebourg a permis l'aménagement de nombreux étangs depuis le Moyen Âge. Les formes d'utilisation de l'eau sont multiples et constituent un patrimoine régional matériel et immatériel. Comme de nombreux pays d'étangs, ce territoire voit évoluer les usages de l'eau. Sa fonction productive, longtemps maintenue dans le contexte de la République Démocratique Allemande, évolue aujourd'hui vers une offre touristique et de loisirs mise en scène par une politique promotionnelle, voire vers un renouveau des usages traditionnels de l'eau. 
Conjointement, les mesures de protection environnementale se développent, notamment dans le cadre de la reconversion des anciennes mines de lignite, ennoyées et aménagées en plans d'eau, transformant ce pays d'étangs en une région de lacs artificiels et d'étangs. La Lusace apparaît ainsi comme un territoire rural renaissant, affirmant une volonté forte de valorisation de ses paysages d'étangs et de son patrimoine culturel.

8 Concernant les pays d'étangs, Raymond Woessner propose, à partir de l'étude de la réalisation de «la Route de la Carpe frite» dans le Sundgau (Haut-Rhin), une analyse sur les enjeux identitaires et territoriaux d'un pays d'étangs. Rappelant la situation marginale du Sundgau des étangs, il évoque les difficultés d'affirmation de ce dernier face aux enjeux des compétences et des découpages territoriaux que l'auteur décrit comme une balkanisation en "micro-territoires». Cette situation fait douter de la capacité du Sundgau à forger un territoire limnique. Toutefois, les enjeux de recompositions liés à la loi Nouvelle Organisation Territoriale de la République (NOTRe) et l'émergence de nouvelles enveloppes territoriales peuvent être un moyen de faire émerger une forme de limno-territorialisation où les étangs pourraient être le catalyseur de l'affirmation d'une identité. C'est ainsi la créativité des acteurs qui permettra de redynamiser une activité déclinante et marginale vouée à la conservation et à la seule préservation des étangs.

Interrogeant les trajectoires des pays d'étangs, Denis Mathis étudie les paysages d'eau du Pays des Mille Étangs en Haute-Saône. Ce pays d'étangs de moyenne montagne est un territoire aménagé par l'homme et destiné à mettre en valeur une activité agrosylvo-pastorale, relayée par les besoins en eau d'activités proto-industrielles et industrielles établies dans les fonds de vallées. Le déclin économique et fonctionnel de ce territoire modifie aujourd'hui ses perceptions. Sa valeur environnementale, écologique et sociale est réaffirmée dans les jeux d'acteurs définissant des projets territoriaux. Toutefois, les dynamiques des paysages et la déprise anthropique fragilisent la conservation des héritages stagnustres et remettent en cause les formes de valorisation d'un patrimoine naturel et humain. Ainsi, la prise en compte de la valeur écologique des espaces d'eau au sein de l'hydrosystème des Mille Étangs doit retenir les formes de complémentarités établies par les anciens systèmes car ces derniers ont construit une gestion cohérente, solidaire et rationnelle de la ressource en eau pour valoriser un territoire et ses périphéries.

10 À l'échelle la plus fine, celle des étangs eux-mêmes, Camille Millot éclaire les incidences de la notion de cours d'eau sur la gestion et la pérennité des étangs à partir de l'aménagement de dérivations. L'utilité de ces dernières dans la gestion permettrait de concilier les étangs et la réglementation et la gestion des cours d'eau. En effet, suivant le présupposé que les étangs impacteraient le bon état écologique des cours d'eau, l'aménagement de dérivation, préconisé au vu de la réglementation des cours d'eau, est considéré comme permettant de limiter ces impacts négatifs. À partir de l'étude de la dérivation de l'étang de Gâte-Souris à Montchevrier (Indre), l'auteure souligne l'intérêt de la dérivation dans le bilan annuel des températures de la Bouzanne. Toutefois, la réalisation de dérivation reste un problème pour les propriétaires et leur imposer cette réalisation a un coût qui peut remettre en cause la pérennité même des étangs et entraîner des stratégies d'effacement.

11 Enfin, Rachid Nedjaï interroge la dynamique hydrosédimentaire des étangs de moyenne montagne au contact des espaces urbain et agricole. Il développe son étude 
sur l'étang de Haute-Jarrie (Isère). Son étude invite à réfléchir sur les enjeux d'étangs désormais inscrits, tout ou partiellement, au sein des espaces urbanisés et dont les fonctionnements sont modifiés par les évolutions du ruissellement et les volumes sédimentaires mobilisés, notamment lors des périodes orageuses. Des actions ont déjà pu être réalisées (curages, plantations), mais ces dernières ont été pour l'heure jugées insuffisantes pour assurer le maintien d'un bon état du plan d'eau. L'auteur s'attache à proposer des solutions pour réduire ces déséquilibres en piégeant une partie des flux sédimentaires (amélioration des pratiques agricoles, modification du sens du labour, utilisation de cultures peu consommatrices d'eau, aménagement de canaux de décantation...). Cette étude permet de souligner la difficile gestion des étangs dans un milieu désormais changeant.

L'ensemble des contributions de ce numéro de la Revue Géographique de l'Est doit permettre d'approfondir l'étude des étangs, mais aussi d'entamer une réflexion sur les trajectoires futures des paysages et pays d'étangs en France et en Europe. Ces dernières ne doivent pas uniquement s'établir au prisme des enjeux qualitatifs de gestion écologique et de préservation ou en tenant compte des éléments sociaux, historiques, culturels et économiques spécifiques aux pays d'étangs et de leurs paysages, mais aussi en tirant hors de leur marginalité ces territoires ruraux pour en faire de véritables pays d'étangs, inscrits dans des dynamiques économiques et sociales pérennes.

\section{BIBLIOGRAPHY}

Bartout P., 2015a, Les territoires limniques. Nouveau concept limnologique pour une gestion géographique des milieux lentiques, Mémoire HDR de géographie, Université d'Orléans, 444 p.

Bartout P., 2015b, « Un potentiel lentique méconnu : la France aux 250000 étangs », in Touchart L., Bartout P., Motchalova O. (dir), Mieux comprendre les étangs : expériences nationales et internationales, du Berry Limousin à l'Europe orientale, Brive-la-Gaillarde, Les Monédières, p. 27-49.

Bartout P., Touchart L., 2013, «L'inventaire des plans d'eau français : outil d'une meilleure gestion des eaux de surface », Annales de Géographie, 691, p. 266-289.

Bédoucha G., 2011, Les liens de l'eau. En Brenne, une société autour des étangs, Paris - Versailles, Maison des Sciences de l'homme et Quæ, 688 p.

Bénarrous R., 2009, La grande Brenne aux périodes préindustrielles : contribution à l'histoire des paysages, des étangs et des relations sociétés/milieux dans une zone continentale : approches historique, archéologique et paléo environnementale, Thèse de doctorat, Université Panthéon Sorbonne de Paris, $605 \mathrm{p}$.

Chiffre E. et Mathis D., 2015, « Prise en compte des hydrosystèmes hérités par les parcs naturels régionaux de Lorraine et du Morvan ", Territoire en mouvement Revue de géographie et aménagement [En ligne], 25-26 | 2015, mis en ligne le 31 mars 2015, URL : http://tem.revues.org/2764

De Carrara St. et Le Lay Y.-F., (2015), « Quand l'usage fait patrimoine. Vers une patrimonialisation des usages et des paysages culturels ? », Développement durable et territoires[En ligne], Vol.6, $\mathrm{n}^{\circ} 1$ | Mars 2015, mis en ligne le 11 février 2016. URL : http://developpementdurable.revues.org/10754 
Mathis D., 2014, « Recomposition territoriale d'un espace rural flou : l'exemple du « Pays des Étangs » en Moselle »,Territoire en mouvement- Revue de géographie et aménagement [En ligne], 22 | 2014, mis en ligne le 15 juin 2014, URL : http://tem.revues.org/2415

Touchart L., Bartout P. et Motchalova O., (dir.), 2015, Mieux comprendre les étangs. Expériences nationales et internationales, Brive, Éditions « les Monédières », 421 p.

Touchart L., (dir.), 2007, Géographie de l'étang. Des théories globales aux pratiques locales, Paris, L'Harmattan, $228 \mathrm{p}$.

\section{AUTHOR}

\section{DENIS MATHIS}

Maître de Conférences en géographie - Université de LorraineLaboratoire d'Observation des TERRitoires (LOTERR)23, boulevard Albert $1^{\mathrm{er}}$ - BP 33-97 - 54015 NANCY CEDEX Tél :

03.54.50.51.27 Mail : denismathis[chez]aol.com 